Trotz dieser Mängel aber ist die Lektüre empfehlenswert für jeden, der sich für die Konvergenz oder Divergenz rechtlicher und politischer Entwicklungen in Afrika interessiert.

Karl Leuteritz

\title{
Ludwik Dembinski
}

The Modern Law of Diplomacy: External missions of states and international organizations

Martinus Nijhoff, Dordrecht/Lancaster/Boston 1988, 282 S., $£ ~ 40,95$

Wie der Untertitel erkennen läßt, faßt Ludwik Dembinski in seiner Abhandlung "The Modern Law of Diplomacy" den Begriff der Diplomatie weiter als bisher üblich und schließt damit eine empfindliche Lücke im völkerrechtlichen Schrifttum: Noch nie war vorher das Recht der Außenvertretungen aller Völkerrechtssubjekte - also der (ständigen) diplomatischen und konsularischen Vertretungen der Staaten untereinander, der Sondermissionen und Delegationen, der Vertretungen von Staaten bei internationalen Organisationen sowie von internationalen Organisationen bei Staaten und untereinander - im Zusammenhang behandelt worden. So macht man sich mit einiger Spannung an die Lektüre und legt das Buch am Ende um wertvolle Erkenntnisse bereichert, wenn auch - wie könnte es bei einem solchen ersten Versuch anders sein - nicht völlig befriedigt, wieder aus der Hand.

Der Verfasser hat sein Werk systematisch gegliedert und behandelt in - zu fünf Teilen zusammengefaßten - 27 Kapiteln zunächst den Gegenstand seiner Untersuchung und die einschlägigen Rechtsgrundlagen (Kap. I und II) und dann nacheinander die Außenvertretungen in ihren verschiedenen Erscheinungsformen, ihre Errichtung, Arbeitsbedingungen und Schließung (Kap. III - IX); das Personal der Vertretungen in seinen verschiedenen Kategorien sowie Beginn und Ende seines Tätigwerdens (Kap. X - XIV); den Status der Vertretungen und ihrer Mitglieder (Kap. XV - XXII); und schließlich die Tätigkeitsbereiche der Außenvertretungen (Kap. XXIII - XXVII). Die Rechtslage wird dabei an Hand der Úbereinkünfte dargestellt, die im wesentlichen aus der Tätigkeit der Wiener Kodifikationskonferenzen seit 1961 hervorgegangen sind: Wiener Übereinkunft über diplomatische Beziehungen von 1961 (WƯD), über konsularische Beziehungen von 1963 (WƯK), über Sondermissionen von 1969 und über die Beziehungen von Staaten mit internationalen Organisationen von 1975. Dazu treten die VN-Abkommen über Vorrechte und Befreiungen der VN und der Sonderorganisationen von 1947, die New Yorker Diplomatenschutzkonvention von 1973 und der Entwurf der "International Law Commission" (ILC) für ein Protokoll über den diplomatischen Kurierbeutel und Kurier. Schrifttum aus der Zeit vor 1960 wird vom Verfasser nicht berücksichtigt. 
Obwohl bisher nur WƯD und WƯK (150 bzw. 117 Teilnehmerstaaten) für die Mehrheit der Völkerrechtsgemeinschaft verbindlich sind, erscheint dieses Verfahren berechtigt: Die Diplomatenschutzkonvention hat es immerhin schon auf 70 Ratifizierungen gebracht, die Übereinkunft über Sondermissionen wurde zwar bisher nur von 29 Staaten ratifiziert, bietet jedoch lediglich Bestimmungen, über die in Praxis und Lehre Einigkeit herrscht, und die Übereinkunft über die Beziehungen von Staaten zu internationalen Organisationen schließlich wird zwar wegen des einhelligen Widerstands der Sitzstaaten gegen die nach ihrer Auffassung überzogenen Vorrechte und Befreiungen wahrscheinlich nie in Kraft treten, bietet aber - von diesem Sondergebiet abgesehen - nur Formulierungen von längst als Gewohnheitsrecht anerkannten Normen.

Die Darstellung ist durchweg klar, übersichtlich und umfassend, was hier nur an einigen Beispielen aufgezeigt werden soll: Im Kap. III wird bei der Darstellung der Vertretungen von Staaten und von intemationalen Organisationen korrekt auf die zwei verschiedenen Formen von EG-Vertretungen (in Industrieländern einerseits und in den AKP-Staaten auf Grund von Art. 128 Lomé III andererseits) hingewiesen. Auch wird den Nuntiaturen und den Vertretungen des Malteser-Ordens (ganze vier, bei Beziehungen zu 41 Staaten!) und des IKRK jeweils ein eigener Absatz gewidmet. Unter der Úberschrift "Mehrfachakkreditierungen" wird neben dem Normalfall der Akkreditierung einer Vertretung in mehreren Ländern (Art. 5 WỦD) auch die Möglichkeit abgehandelt, gemeinsame Vertretungen mehrerer Staaten in einem Empfängerstaat (Art. 6 WUD) zu errichten, wie dies jetzt erstmals mit deutsch-französischen Botschaften in Ulan Bator (Mongolei) und Gaborone (Botswana) praktiziert werden soll. In Kap. VI wird klar herausgearbeitet, daß Sondervertretungen im Bereich der wirtschaftlichen Zusammenarbeit (US-AID, UNDP, EG in AKPStaaten) im Gegensatz zum sonst geltenden Gebot der Nichteinmischung in die inneren Verhältnisse ihre Hauptaufgabe gerade in der Mitgestaltung der Verhältnisse des Empfängerlandes haben und zwar auch dann, wenn diese Aufgaben einem Mitglied der "normalen" diplomatischen Vertretung des Entsendestaates übertragen sind.

Auf die deutliche Betonung des Konsensualprinzips und seiner Auswirkungen bei der Arbeitsaufnahme und -beendigung der Vertretungen selbst, ihrer Leiter und ihres Personals (Kap. VII, IX, XIII und XIV) sei nur kurz hingewiesen. Von größter praktischer Bedeutung dürften aber die Statusfragen behandelnden Kapitel XV bis XXII sein. Hier stellt der Verfasser zu Recht auf das notwendige Gleichgewicht der Rechte und Pflichten von Entsender und Empfänger ab. Der Schutzpflicht des Empfängers gegenüber der Vertretung, deren Kommunikationsfreiheit, den Grundsätzen der Gewährung von Vorrechten und Befreiungen, der Unverletzlichkeit, der Befreiung von der Gerichtsbarkeit, der Steuer- und Zollbefreiung und der Beilegung von Streitfällen ist je ein Kapitel gewidmet. Nach geltendem Recht ist hier alles klar. Daß es trotzdem in der Praxis zu Schwierigkeiten kommen kann, dafür ein selbst erlebtes Beispiel: Monatelang verhandelte ein Gericht in einem WUK-Partnerland die Zivilklage eines seiner Staatsangehörigen gegen einen deutschen Konsularbeamten, obwohl dieser sich sofort auf seine Befreiung von der Gerichtsbarkeit nach Art. 43 Abs.1 WUK berufen hatte, da alle ihm vorgeworfenen Handlungen unstreitig konsulari- 
sche Amtshandlungen gewesen waren. Es ergab sich, daß das Wort "Handlungen" in Art. 43 WUK bei der Úbersetzung in die Landessprache mit einem Terminus wiedergegeben worden war, der "strafbare Handlungen" bedeutete und das Gericht deshalb in der Berufung auf diese Vorschrift die Selbstbezichtigung des Beklagten sah, eine Straftat begangen zu haben! Das Verfahren wurde schließlich eingestellt, weil der Kläger den Anspruch nicht weiter verfolgte. Eine Klärung der Rechtsfrage erfolgte nicht.

Bei der Behandlung der Tätigkeiten der Vertretungen (Kap. XXIII - XXVI) vertritt der Verfasser insoweit eine zu enge Auffassung, als er das Gebot der Nichteinmischung in die inneren Verhältnisse (Art. 41 WUD, 55 WUK u.a.) auch auf die Außenpolitik des Empfängerstaates bezieht, was allerdings "früher" nicht einhellige Auffasung gewesen sei: Jeder Versuch der Vertretung, diese zu beeinflussen, stelle einen Angriff auf die Souveränität dar. Die so postulierte Beschränkung auf reine Informationsaufgaben führt zu einem für die diplomatische Praxis absurden Ergebnis. Die als "Beleg" zitierte Abberufung des deutschen Botschafters Grewe aus Washington 1961 (in Wirklichkeit 1962) wegen "öffentlicher Kommentare zur US-Außenpolitik" (S. 232) taugt hierzu nicht: Weder hatte der Botschafter solche Kommentare abgegeben, noch wurde er deswegen abberufen. Sein Weggang war vielmehr die politische (nicht rechtliche) Konsequenz aus einer Kontroverse zwischen beiden Regierungen über die Deutschland- und Berlinpolitik der USA. Diese aber auf diplomatischem Wege zu beeinflussen mußte eine Hauptaufgabe der Botschaft sein!

Das Schlußkapitel (XXVII) schließlich trägt eine mißverständliche Úberschrift: Mit "The Rules of Parliamentary Diplomacy" sind nicht die internationalen Aktivitäten der Parlamente und ihrer Abgeordneten gemeint, sondern die Regeln der Konferenzdiplomatie nach (quasi) parlamentarischen Verfahren.

Leider hat ein spezialisierter Druckfehlerteufel häufig "of" statt "or" gesetzt, was beim Lesen irritiert. Eher ein Denkfehler ist es, wenn der Berliner Kongreß auf 1886 statt auf 1878 angesetzt wird (S. 255), und ausgesprochen ärgerlich, wenn die EG durchweg nur als "EEC" (EWG) auftreten und die Bundesrepublik Deutschland ebenso durchgängig als "German Federal Republic" bezeichnet wird.

Vielleicht hängt dies damit zusammen, daß der Verfasser keine deutschsprachige Literatur benutzt hat: Das Literaturverzeichnis enthält - in der Folge der Häufigkeit - Werke in englischer, französischer, italienischer, spanischer, niederländischer und polnischer Sprache. Der Verfasser des einen polnischen Beitrags ist sogar mit zehn Titeln (meist englisch oder französissch) der meistzitierte Autor überhaupt (Franciszek Przetacznik). Neben der deutschen fehlt auch die russische bzw. sowjetische Literatur völlig, obwohl dort sicher einiges zum Thema geschrieben wurde.

Trotz der aufgezeigten Mängel gehört das Buch in jedes völkerrechtliche Seminar und in die Hand eines jeden, der sich theoretisch oder praktisch mit internationalen Beziehungen befaßt: Nicht als Bibel oder Glaubensbekenntnis, aber als nützliche Informationsquelle und anregende Diskussionsgrundlage.

Karl Leuteritz 